\title{
Effects of signal diffusion on $x$-ray phase contrast images
}

K. Ignatyev, P. R. T. Munro, R. D. Speller, and A. Olivo

Citation: Review of Scientific Instruments 82, 073702 (2011);

View online: https://doi.org/10.1063/1.3606442

View Table of Contents: http://aip.scitation.org/toc/rsi/82/7

Published by the American Institute of Physics

\section{Articles you may be interested in}

Method for automatization of the alignment of a laboratory based $x$-ray phase contrast edge illumination system Review of Scientific Instruments 84, 083702 (2013); 10.1063/1.4816827

Sensitivity of laboratory based implementations of edge illumination X-ray phase-contrast imaging Applied Physics Letters 103, 244104 (2013); 10.1063/1.4845015

Coded apertures allow high-energy $x$-ray phase contrast imaging with laboratory sources Journal of Applied Physics 110, 014906 (2011); 10.1063/1.3605514

Compact and cost effective lab-based edge-illumination $x$-ray phase contrast imaging with a structured focal spot Applied Physics Letters 108, 224102 (2016); 10.1063/1.4953459

Laboratory-based X-ray phase-contrast imaging with misaligned optical elements Applied Physics Letters 107, 124103 (2015); 10.1063/1.4931778

A laboratory based edge-illumination $\mathrm{x}$-ray phase-contrast imaging setup with two-directional sensitivity Applied Physics Letters 107, 204105 (2015); 10.1063/1.4935983

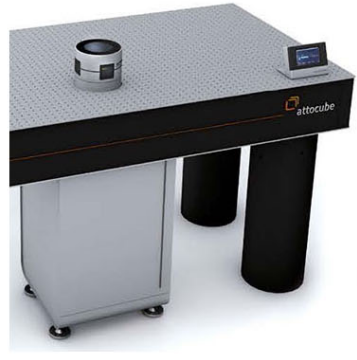

Obstruction free access optical table with integrated cryocooler

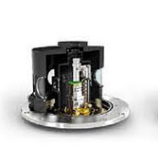

Various Objective 0ptions

\section{attoDRY800}

- Cryogenic Temperatures

- Ultra-Low Vibration

- Optical Table Included

- Fast Cooldown

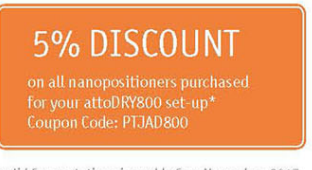




\title{
Effects of signal diffusion on $\mathrm{x}$-ray phase contrast images
}

\author{
K. Ignatyev, P. R. T. Munro, R. D. Speller, and A. Olivo \\ Department of Medical Physics and Bioengineering, UCL, Malet Place, Gower Street, \\ London WCIE 6BT, United Kingdom
}

(Received 9 May 2011; accepted 8 June 2011; published online 8 July 2011)

\begin{abstract}
We discuss the problem of signal diffusion among neighbouring pixels in $\mathrm{x}$-ray phase contrast imaging (XPCi) specifically for coded-aperture (CA) XPCi, but many of the discussed observations are directly transferable to other XPCi modalities. CA XPCi exploits the principle of pixel edge illumination by means of two CA masks. The first mask, placed in contact with the detector, creates insensitive regions between adjacent pixels; the second one, placed immediately before the sample, creates individual beams impinging on the boundaries between sensitive and insensitive regions on the detector, as created by the detector mask. In this way, edge illumination is achieved for all pixels of an area detector illuminated by a divergent and polychromatic beam generated by a conventional source. As the detector mask redefines the resolution properties of the detector, sample dithering can be used to effectively increase the system spatial resolution, without having to apply any post-processing procedure (e.g., deconvolution). This however creates artifacts in the form of secondary fringes (which have nothing to do with phase-related secondary fringes) if there is signal diffusion between adjacent pixels. In non-dithered images, signal diffusion between adjacent pixels causes a reduction in image contrast. This effect is investigated both theoretically and experimentally, and its direct implications on image quality are discussed. The interplay with the sample positioning with respect to the detector pixel matrix, which also has an effect on the obtained image contrast, is also discussed. (c) 2011 American Institute of Physics. [doi:10.1063/1.3606442]
\end{abstract}

\section{INTRODUCTION}

In $\mathrm{x}$-ray phase contrast imaging (XPCi), image contrast arises from refraction/interference effects, instead of absorption. This leads to significant image enhancements: increased visibility of all details, and possible detection of features classically considered invisible. ${ }^{1-5}$ As a consequence of this, a multitude of different applications have been proposed - primarily in medical imaging, ${ }^{4-12}$ including in vivo, ${ }^{12}$ but also in fields as diverse as material science, ${ }^{13-16}$ cultural heritage preservation, ${ }^{17,18}$ homeland security, ${ }^{19}$ and others.

However, so far these applications remained restricted to the research arena, and an effective translation into realworld applications has yet to be achieved, with the partial, albeit notable exception of the in vivo mammography study underway in Trieste, ${ }^{12}$ which can however still be considered primarily a research exercise. Partial exceptions are also the mammography system commercialized by Konica/Minolta, which, however, did not provide a significant difference in recall and cancer detection rates,${ }^{20}$ possibly due to moderate phase-contrast enhancement, and commercial systems based on microfocal sources, typically requiring long exposure times. ${ }^{21,22}$

This is due to XPCi being particularly demanding in terms of source characteristics, particularly spatial coherence. As a consequence of this, until recently almost all experiments were either based on synchrotron radiation (SR), or on microfocal $\mathrm{x}$-ray sources. ${ }^{3,23}$ The problem with the former is that of restricted accessibility and excessive cost, while the use of the latter results in exposure times too long to be of use in many real-world applications, due to limited x-ray output. Crystalbased methods can provide an exception and be implemented with conventional sources, ${ }^{2,24}$ but at the cost of an increased exposure time due to the strong selection on the emitted $\mathrm{x}$-ray spectrum operated by the crystal itself.

Grating-based XPCi techniques ${ }^{25-27}$ have been proposed as a solution to this problem. In this method, two sets of gratings are introduced between sample and detector - these can either be a "phase" (i.e., low absorbing) and an absorption grating, or two absorption gratings. The two gratings are separated by a Talbot distance, ${ }^{28}$ so that a self-image of the first grating is projected onto the second one. One grating is then scanned with respect to the other, orthogonally to the grating lines, and a series of images is acquired at different grating positions ("phase stepping"). The presence of a sample distorts the approximately sinusoidal signal obtained in this way, and analyzing such distortions allows absorption, phase and dark-field signals to be independently measured.

As gratings typically have a pitch of a few $\mu \mathrm{m}$, the method is still highly sensitive to spatial coherence, as a projected source size comparable to the grating pitch has the result of mixing minima and maxima together, i.e., flattening out the above sinusoidal wave, thus making phase signals undetectable. However, relaxed requirements were demonstrated in terms of temporal coherence, ${ }^{29}$ as an energy bandwidth increase of up to 5\%-10\% has the result of reducing the peak-to-peak difference in the above sinusoidal signal, without however making it disappear.

Based on these ideas, the method was translated into use with conventional sources by introducing a third grating, placed in contact with the x-ray source, which generates an array of mutually incoherent, but individually coherent sources $^{30}$ (i.e., switching form the Talbot to the Talbot-Lau 
configuration). Like previous XPCi approaches, if the third "source" grating is not used, then the method still requires either SR or a microfocal source ${ }^{31}$ in order to work.

We discuss here a different approach, in which nonmicrofocal sources with focal spots of up to $100 \mu \mathrm{m}$ (Ref. 32) can be used without additional aperturing or collimation. The method adapts the "pixel edge illumination" concept developed in an earlier SR experiment ${ }^{33}$ to a divergent, polychromatic source by means of two sets of coded apertures (CA), one placed immediately before the object to be imaged, and the other placed in contact with the detector. ${ }^{32,34,35}$ The apertures have a pitch equal to that of the detector pixels (50-100 $\mu \mathrm{m}$ ), consequently relaxing the requirements on the spatial coherence. ${ }^{32}$ Even though the set-up might present some similarities with the Talbot interferometry, as they both employ grating-like objects, the way in which the system is made sensitive to phase effects is intrinsically different. ${ }^{36,37}$

Both Talbot interferometry and CA XPCi are affected by signal diffusion between adjacent pixels. This can be expressed as the proportion of signal counted by the neighbouring pixels when only a given pixel is illuminated by x-rays, and is primarily due to two effects - charge sharing between adjacent detector pixels, and light diffusion in the scintillator. The latter is by far the dominating factor in indirect detection systems, although also the former has a non-negligible effect, as demonstrated by the fact that some direct conversion system have a non-zero signal diffusion coefficient. ${ }^{38}$

This paper deals with the effect that such signal "spillout" has on image quality and detected contrast in CA XPCi, through simulation studies and direct experimental verification of the same. It can be easily understood that, as the phase sensitivity in CA XPCi depends on illuminating only the transition region between sensitive and insensitive areas on a pixel, inducing signal in the adjacent pixel will make this transition less sharp, thus affecting the sensitivity, and consequently reducing the detected contrast for a given object.

This also has an additional effect on "dithered" image acquisitions. With "dithering," we refer here to the acquisition of a series of images at sub-pixel sample displacements. These sub-images are then recombined to obtain an image in which the resolution is determined by the dithering step, rather than by the pixel size. Normally, in order to obtain such resolution enhancement, a post-processing procedure (deconvolution) is required. ${ }^{39,40}$ In the case of CA XPCi, however, the active surface of the detector pixel, and consequently its point spread function, are redefined (namely, narrowed down) by the detector CA mask. Therefore, as long as the dithering step is larger than or equal to the aperture size in the detector mask, the deconvolution procedure is not necessary, and an image with increased resolution is obtained as a direct result of the appropriate recombination of the dithered sub-images. However, if signal spill-out is present, this process generates image artifacts, which is one of the items discussed below.

Likewise, it is reasonable to expect that signal spill-out would affect also grating interferometry (e.g., by reducing fringe visibility), and therefore the present study might have some relevance, or at least trigger further investigations, also in that, and possibly other, XPCi areas. Overall, it can be concluded that detector systems based on the direct conver- sion technology would appear the right choice surely for CA $\mathrm{XPCi}$ and possible also for other XPCi approaches, although if signal spill-out is not excessive (e.g., in indirect conversion system based on the direct deposition of columnar CsI), the side effects caused by it could be considered acceptable in many cases. Moreover, there are strategies that can be adopted to mitigate its negative effects, which are also discussed in Secs. II and IV.

\section{MATERIALS AND METHODS}

A CA XPCi imaging system was designed and assembled along the lines described in Ref. 38. A rotary target, small-focused W source, used in hospital work since the mid-eighties ${ }^{41}$ was used. Its focal spot was determined to be approximately $50 \mu \mathrm{m}$ by measuring the penumbra caused by an edge. The edge was placed at a series of different source/edge/detector distance combinations and the same result for the source size was consistently found. The Hamamatsu C9732DK flat panel detector, a passive-pixel CMOS sensor with a pixel size of $50 \mu \mathrm{m}$, was used as the detector. This is an indirect conversion detector system, with x-ray conversion obtained in a directly deposited, $160 \mu \mathrm{m}$ thick structured CsI layer. The use of such a device results in a $49 \%$ and $6 \%$ signal spill-out on the first and second neighbour, respectively, when only one pixel is illuminated. As we are here dealing with a system sensitive to phase effects in one direction only, this was measured by illuminating only one pixel with a "blade" of radiation smaller than the pixel side in one direction, and observing the intensity induced in the (non-illuminated) first and second neighbours. ${ }^{38}$ In the case of a system sensitive to phase effects in two directions, a pencil beam (with a cross-section smaller than the pixel) would have to be used, and the induced intensity measured in both directions.

The source-to-detector distance was $2 \mathrm{~m}$ and the sourceto-sample distance $1.6 \mathrm{~m}$.

While results previously presented ${ }^{37}$ were obtained with a detector CA mask with an aperture pitch of $100 \mu \mathrm{m}$ ("column-skipping"), in the experiment discussed here a mask with a pitch equal to that of the detector pixels (50 $\mu \mathrm{m}$, "non-column-skipping") was used, to investigate the effects of signal diffusion in detail. Previously, the aim was to maximize the phase contrast signal while using an indirect conversion detector: skipping every second line meant that, overall, the signal spill-out was effectively of $\sim 6 \%$, and therefore practically negligible. In this case, the spill-out of $49 \%$ had a significant effect on the acquired images, as discussed below. The difference between the two set-ups is schematized in Fig. 1.

The pitch in the detector mask was actually slightly reduced with respect to the pixel pitch (i.e., 48 vs. $50 \mu \mathrm{m}$ ), to allow for some distance between detector mask and detector surface, and therefore for an easier alignment. The apertures in the detector mask were of $15 \mu \mathrm{m}$. The pre-sample mask was a replica of the detector mask, scaled down to account for the beam divergence considering a distance between the two masks of approximately $40 \mathrm{~cm}$, i.e., the pitch was $38 \mu \mathrm{m}$ and the apertures $12 \mu \mathrm{m}$. Both masks were manufactured to the 

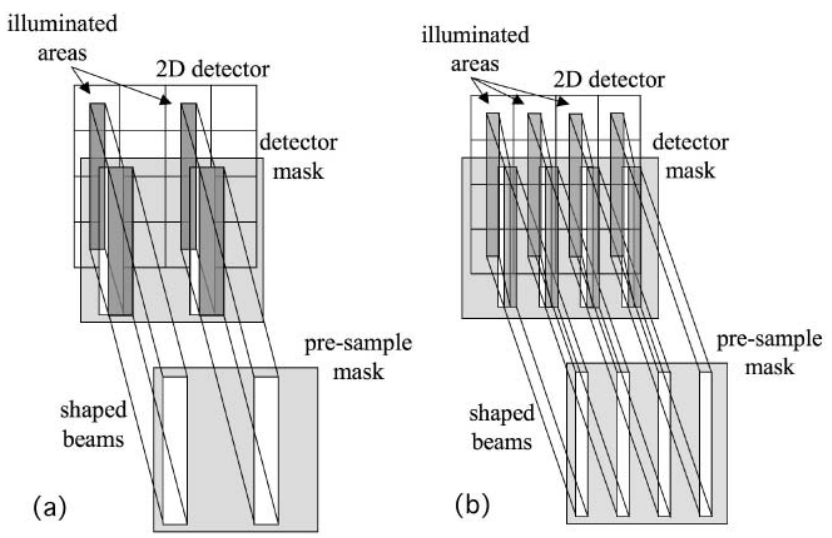

FIG. 1. Schematic of the column-skipped (a) and non-column-skipped (b) CA XPCi set-up. In both cases the set-up is shown from the pre-sample CA mask to the detector, as only the $\mathrm{x}$-ray source appears further upstream. The beam is unshaped and uncollimated before it impinges on the pre-sample CA mask, and the sample is placed immediately downstream of such mask. The illuminated regions on the detector and the detector mask are shown as darker shades of grey. The apertures in the detector mask effectively redefine the pixel surface, and as a consequence the apertures themselves are "edgeilluminated" by the shaped beams.

authors design by Creatv Microtech (Potomac, MD) by gold electroplating on a patterned graphite substrate, for an overall gold thickness of about $80 \mu \mathrm{m}$. Both masks were mounted on micrometric precision alignment systems (Newport Microcontrole translators and Kohzu cradles), and the samples were also mounted on a Newport translation stage for sample dithering.

The simulated results were obtained through a raytracing based Monte Carlo type program in which x-rays are generated at a given position (the "source"), travel through the first CA mask and are either absorbed or transmitted. If transmitted, they interact with the sample, and have their direction consequently changed if appropriate. They then travel through the second CA mask and, if they hit one of the apertures in this, increase the number of counts in the appropriate detector pixel. All parameters (source-to-sample and sample-todetector distance, detector pitch, sample and detector masks pitch and apertures size, etc.) can be varied. A detailed description of the simulation can be found in Ref. 32. Extended sources are simulated by generating patterns created by point sources displaced in different points in space, and then by creating a weighted average of such profiles on the basis of the source distribution. The beam polychromaticity is dealt with according to the procedure outlined in Refs. 42 and 32. Signal spill-out can be implemented either by "injecting" the appropriate amount of induced signal in the adjacent pixels, ${ }^{32}$ or by convolving the individual profiles unaffected by spill-out with the appropriate spread function.

\section{RESULTS}

Figure 2 shows the effect of $49 \%$ signal spill-out between adjacent pixels on simulated CA XPCi profiles of a $300 \mu \mathrm{m}$ thick polyethylene fibre (experimental setting as described above, with $40 \mathrm{kVp}$ tube potential). These profiles are nondithered, i.e., the sample is kept still in a given position and
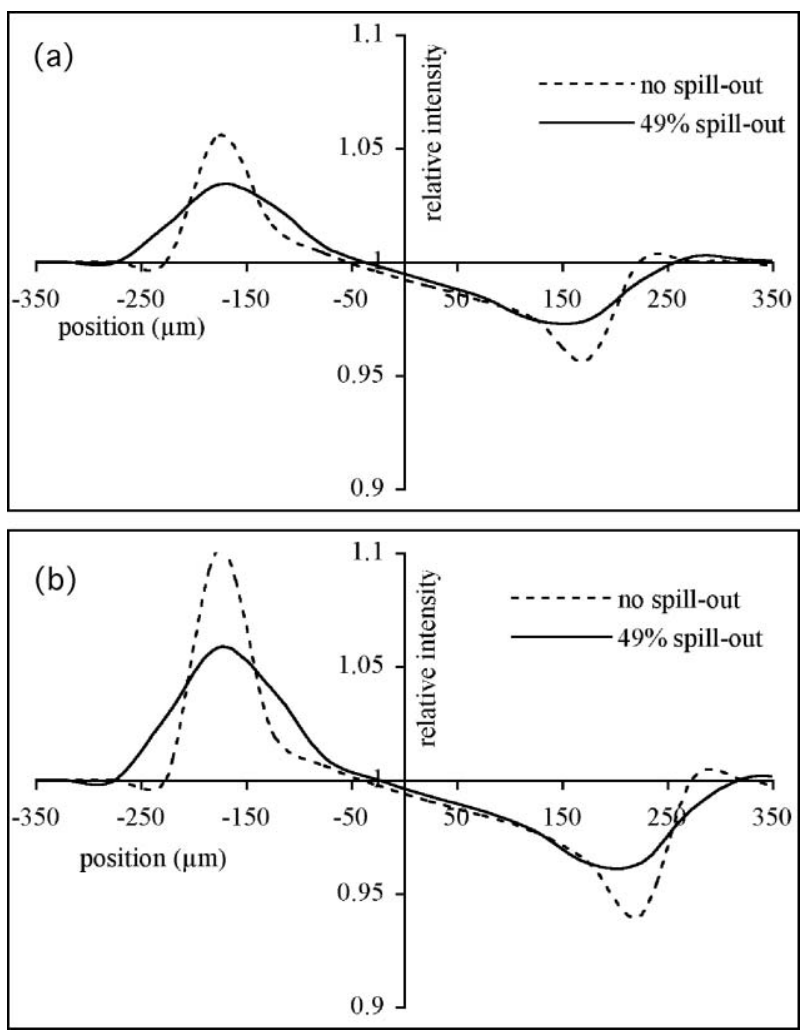

FIG. 2. Simulated profiles for non-dithered CA XPCi profiles of a $300 \mu \mathrm{m}$ thick polyethylene fibre, with (solid lines) and without (dashed lines) the effect of $49 \%$ signal spill-out between adjacent pixels. The difference between the graphs shown in (a) and (b) is due to a sub-pixel difference in the sample position.

a single image is acquired. In this case, as it can be seen the effect of signal spill-out is simply that of smearing out the profiles, consequently reducing the peak-to-peak difference, i.e., the phase contrast.

It should be noted that the two profiles shown in Figs. 2(a) and 2(b) were obtained for exactly the same sample in the same experimental conditions, the only difference being a small (sub-pixel) shift of the sample position. As it can be seen, as a consequence of this the peak-to-peak difference changes substantially, both in the "spill-out affected" and "non-affected" profiles. This is hardly surprising as most of the signal is generated by the sample edge; therefore, the

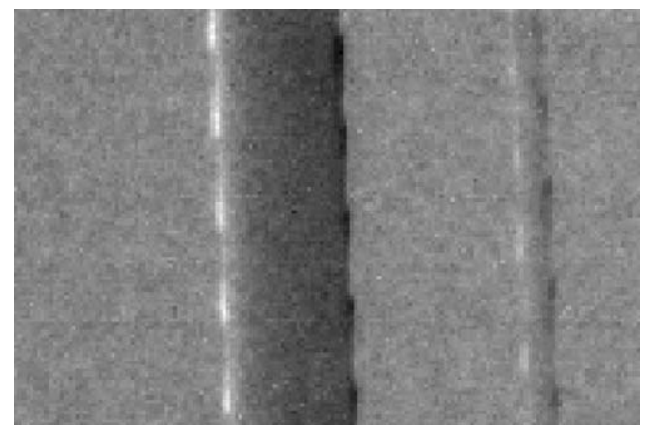

FIG. 3. Experimental undithered CA XPCi image of two polyethylene fibres -1300 (left) and 300 (right) $\mu \mathrm{m}$ in diameter. Spatial resolution limits cause a periodically varying intensity of the XPCi fringes on the edges of the fibres (see text). 
relative position of sample and detector pixel edges has a substantial impact on the detected contrast.

This can be clearly appreciated in the non-dithered experimental CA XPCi image shown in Fig. 3, showing a thick $(1.3 \mathrm{~mm}$, on the left-hand side) and a thin $(300 \mu \mathrm{m}$, righthand side) polyethylene fibre imaged with a tube potential of $40 \mathrm{kVp}$. Both fibres are slightly inclined with respect to the detector pixel columns, and therefore every image line sees the samples in a slightly different position. As a consequence of this, the intensity of both the positive (on the left-hand side of each fibre) and the negative (on the right-hand side) peaks varies going from the top to the bottom of the image. Depending on the inclination of the fibres, after a given number of rows the sample will present itself to the pixel array in the same relative position, apart from the skipping of one pixel -
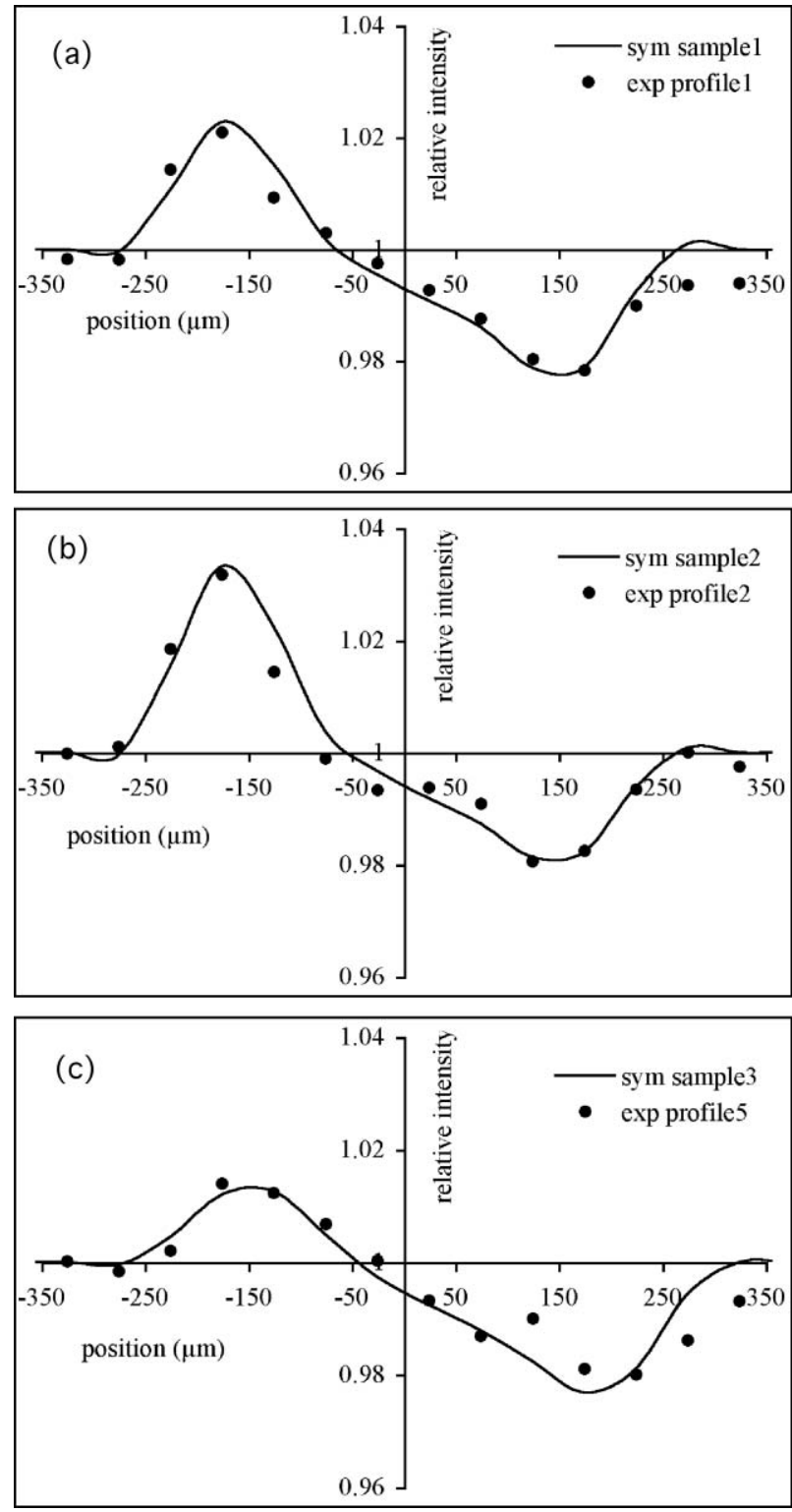

FIG. 4. Comparison between experimental (dots) and simulated (solid lines) profiles of undithered CA XPCi images of a $300 \mu \mathrm{m}$ thick polyethylene fibre. The experimental profiles corresponds to lines extracted from Fig. 3 at different heights, and the solid lines were simulated by varying the sub-pixel displacement of the sample accordingly.

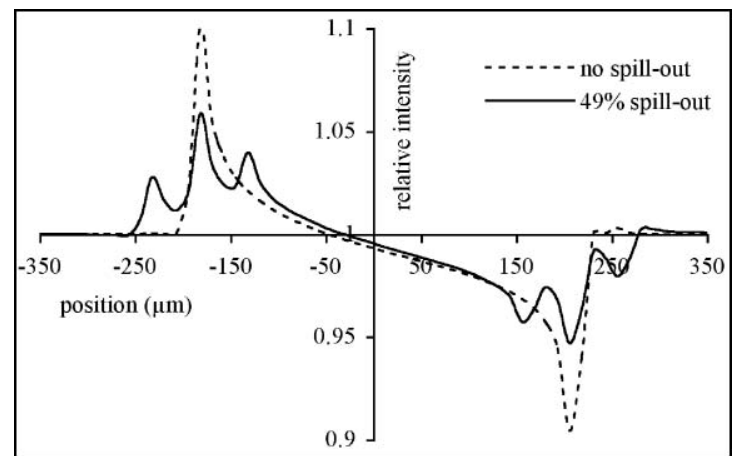

FIG. 5. Simulated profiles for dithered CA XPCi profiles of a $300 \mu \mathrm{m}$ thick polyethylene fibre, with (solid line) and without (dashed line) 49\% signal spill-out between adjacent pixels. In this case, in addition to the signal reduction, spurious peaks appear as a consequence of signal spill-out (see text).

and in fact the intensity variation repeats itself periodically over the image. Figure 4 shows three different experimental profiles of the thin fibre extracted at different heights in the image (i.e., different image rows), and these are compared to simulated profiles obtained in the same experimental condition but changing the sample position by small, sub-pixel steps. The three experimental profiles were extracted randomly, and 10 simulated profiles were created by shifting the sample position by $1 / 10$ of the pixel dimension (scaled down to account for the sample magnification). The best matches were then associated by chi square minimization. As it can be seen, a good agreement was obtained in all three cases between simulation and experiment, validating the explanation of the local peak-to-peak fluctuations.

The concept of "dithering" entails combining profiles like those shown in Fig. 4 in an orderly fashion. In the absence of signal spill-out, this can be done without creating any image artifact. If non-negligible spill-out is present, however, further complications may occur. For example, it can happen that, in a given sample position, the peak intensity $\left(P_{1}\right)$ is so much higher than that of the peak of the next sample position $\left(P_{2},\right)$ that the signal induced in the adjacent pixels is higher than $P_{2}$ itself. If this happens, when the profiles corresponding to the various sample positions are recombined to form the image with increased resolution, spurious oscillations are observed around the "primary" peak. This is shown in the simulated dithered profiles of Fig. 5, where again signals affected and not affected by signal spill-out are compared. In addition to the reduction in the peak-to-peak difference, analogous to

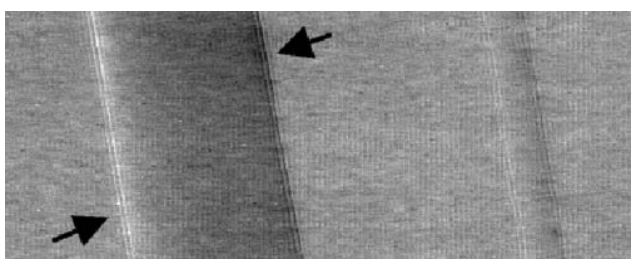

FIG. 6. Experimental dithered CA XPCi image of two polyethylene fibres -1300 (left) and 300 (right) $\mu \mathrm{m}$ in diameter. Additional fringes can be observed along the edges of each fibre, which are an artifact caused by signal spill-out and do not have a phase nature. 


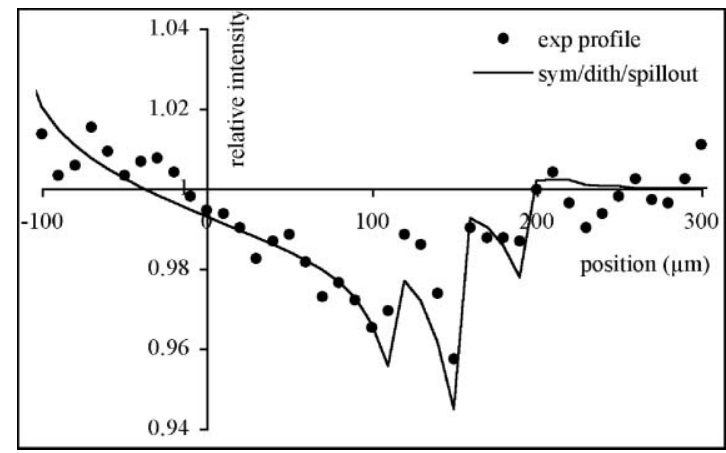

FIG. 7. Comparison between experimental (dots) and simulated (solid line) profiles of dithered CA XPCi images of a $300 \mu \mathrm{m}$ thick polyethylene fibre. The experimental profile is extracted from Fig. 6. As it can be seen, the trend of the multiple peaks is correctly reproduced by the simulation.

that observed in Fig. 2, additional spurious peaks can be observed in the profile affected by (49\%) signal spill-out.

This was verified experimentally by acquiring a dithered image of the sample shown in Fig. 3. Five images like the one shown in Fig. 3 were acquired while moving the sample by $8 \mu \mathrm{m}$ after each image. Considering the acquisition geometry (source-to-sample distance $=1.6 \mathrm{~m}$ and sample-to-detector distance $=0.4 \mathrm{~m}$ ), and the $50 \mu \mathrm{m}$ pixel size, this practically corresponds to subdividing the pixel into five identical (10 $\mu \mathrm{m})$ steps. The resulting images were recombined and the result is shown in Fig. 6.

Upon close inspection, "secondary" fringes can be clearly seen running along the edges of both fibres (see arrows in Fig. 6). It is important to note that these fringes have nothing to do with "phase" related secondary fringes: they are instead an artifact caused by the peculiar acquisition conditions described above, and in particular by the presence of a strong signal spill-out between adjacent pixels. Figure 7 shows the comparison between simulated and experimental profiles of a dithered image, and, even though the agreement is not as striking as that of Fig. 4 (the creation of multiple profiles at even finer sampling steps and subsequent matching through chi-square minimization was not performed in this case), the shape, distribution and trend of the multiple peaks is clearly reproduced.

The presence of these spurious fringes could create a problem in that they might limit the extent to which a resolution increase can effectively be obtained by means of the dithering method, and to avoid this one would have to deconvolve the individual images before recombining them. The extent to which this problem would manifest would clearly depend on the imaged sample, and in particular on the sharpness of the transition between regions with different refractive indices.

\section{CONCLUSIONS}

We have investigated in detail the effect that signal spill-out has on image contrast in XPCi. This paper deals specifically with CA XPCi, but the similarity in the shape of the acquired differential phase contrast signal suggests that similar effects could be encountered also in other XPCi modalities (e.g., grating and crystal-based). Signal spill-out has the effect of reducing image contrast, and the interplay with contrast variations associated with sample positioning at the sub-pixel level gives rise to image artifacts when resolution-enhancing methods like sample dithering are applied. As a byproduct of the above investigation, variations in the degree of phase contrast due to sub-pixel sample positioning were also discussed which would again be relevant also to other XPCi methods.

The analysis indicates direct conversion systems as most appropriate for CA XPCi, and possibly for XPCi in general: ideally, the full-width at half maximum of the detector point spread function should be smaller or equal to the pixel size (and in fact the mammography system currently under development in our labs ${ }^{43}$ will be based on the ANRAD amorphous selenium direct conversion flat panel). However, alternative solutions are offered by pixel skipping strategies, or, alternatively, a small reduction in image contrast could be accepted, depending on the specific application, if the signal spill-out is not too large.

\section{ACKNOWLEDGMENTS}

A. Olivo is supported by a Career Acceleration Fellowship awarded by the UK Engineering and Physical Sciences Research Council (EP/G004250/1).

${ }^{1}$ A. Snigirev, I. Snigireva, V. Kohn, S. Kuznetsov, and I. Schelokov, Rev. Sci. Instrum. 66, 5486 (1995).

${ }^{2}$ V. N. Ingal and E. A. Beliaevskaya, J. Phys. D: Appl. Phys. 28, 2314 (1995).

${ }^{3}$ T. J. Davis, D. Gao, T. E. Gureyev, A. W. Stevenson, and S. W. Wilkins, Nature (London) 373, 595 (1995).

${ }^{4}$ A. Momose, T. Takeda, Y. Itai, and K. Hirano, Nat. Med. 2, 473 (1996).

${ }^{5}$ F. Arfelli, M. Assante, V. Bonvicini, A. Bravin, G. Cantatore, E. Castelli, L. Dalla Palma, M. Di Michiel, R. Longo, A. Olivo, S. Pani, D. Pontoni, P. Poropat, M. Prest, A. Rashevsky, G. Tromba, A. Vacchi, E. Vallazza, and F. Zanconati, Phys. Med. Biol. 43, 2845 (1998).

${ }^{6}$ E. D. Pisano, R. E. Johnson, D. Chapman, J. Geradts, M. V. Iacocca, C. A. Livasy, D. B. Washburn, D. E. Sayers, Z. Zhong, M. Z. Kiss, and W. C. Thomlinson, Radiology 214, 895 (2000).

${ }^{7}$ K. Mori, H. Sato, N. Sekine, N. Shikano, M. Sato, D. Shimao, T. Igarashi, H Shiwaku, and H. Hyodo, Anal. Sci. 17, 1427 (2001).

${ }^{8}$ T. Takeda, A. Momose, J. Wu, Q. Yu, T. Zeniya, T. Lwin, A. Yoneyama, and Y. Itai, Circulation 105, 1708 (2002).

${ }^{9}$ M. J. Kitchen, D. Paganin, R. A. Lewis, N. Yagi, K. Uesugi, and S. T. Mudie, Phys. Med. Biol. 49, 4335 (2004).

${ }^{10}$ A. Wagner, M. Aurich, N. Sieber, M. Stoessel, W. D. Wetzel, K. Schmuck, M. Lohmann, B. Reime, J. Metge, P. Coan, A. Bravin, F. Arfelli, L. Rigon, R. H. Menk, G. Heitner, T. Irving, Z. Zhong, C. Muehleman, and J. A. Mollenhauer, Nucl. Instrum. Methods A 548, 47 (2005).

${ }^{11}$ D. M. Connors, D. Sayers, D. R. Sumner, and Z. Zhong, Phys. Med. Biol. 51, 3283 (2006)

${ }^{12}$ D. Dreossi, A. Abrami, F. Arfelli, P. Bregant, K. Casarin, V. Chenda, M. A. Cova, R. Longo, R. H. Menk, E. Quai, E. Quaia, L. Rigon, T. Rokvic, D. Sanabor, M. Tonutti, G. Tromba, A. Vascotto, F. Zanconati, and E. Castelli, Eur. J. Radiol. 68S, S58 (2008).

${ }^{13}$ P. Cloetens, M. Pateyron-Salomé, J. Y. Buffiere, G. Peix, J. Baruchel, F. Peyrin, and M. Schlenker, J. Appl. Phys. 81, 5878 (1997).

${ }^{14}$ Z. W. Hu, P. A. Thomas, A. Snigirev, I. Snigireva, A. Souvorov, P. G. R. Smith, G. W. Ross, and S. Teat, Nature (London) 392, 690 (1998).

${ }^{15}$ R. H. Mathiesen, L. Arnberg, K. Ramsøskar, T. Weitkamp, C. Rau, and A. Snigirev, Metall. Mater. Trans. B 33, 613 (2002).

${ }^{16}$ A. W. Stevenson, T. E. Gureyev, D. Paganin, S. W. Wilkins, T. Weitkamp, A. Snigirev, C. Rau, I. Snigireva, H. S. Youn, I. P. Dolbnya, W. Yun, B. Lai, R. F. Garrett, D. J. Cookson, K. Hyodo, and M. Ando, Nucl. Instrum. Methods B 199, 427 (2003). 
${ }^{17}$ M. L. Young, F. Casadio, S. Schnepp, J. Almer, D. R. Haeffner, and D. C. Dunand, Appl. Phys. A 83, 163 (2006).

${ }^{18}$ K. Krug, L. Porra, P. Coan, A. Wallert, J. Dik, A. Coerdt, A. Bravin, M. Elyyan, P. Reischig, L. Helfen, and T. Baumbach, J. Synchrotron Radiat. 15, 55 (2008).

${ }^{19}$ A. Olivo, D. Chana, and R. Speller, J. Phys. D: Appl. Phys. 41, 225503 (2008).

${ }^{20}$ T. Morita, M. Yamada, A. Kano, A. Nagatsuka, C. Honda, and T. Endo, in Proceedings of the International Workshop on Digital Mammography 2008, edited by E. A. Kupinsky (Springer-Verlag, Berlin, 2008), pp. $48-54$.

${ }^{21}$ See http://www.xrt.com.au/.

${ }^{22}$ See http://www.xradia.com/.

${ }^{23}$ S. W. Wilkins, T. E. Gureyev, D. Gao, A. Pogany, and A. W. Stevenson, Nature (London) 384, 335 (1996).

${ }^{24}$ D. J. Vine, D. M. Paganin, K. M. Pavlov, J. Kräusslich, O Wehrhan, I. Uschmann, and E. Förster, Appl. Phys. Lett. 91, 254110 (2007).

${ }^{25}$ J. F. Clauser "Ultrahigh resolution interferometric x-ray imaging" U.S. patent 5,812,629 (22 September 1998).

${ }^{26}$ C. David, B. Nöhammer, H. H. Solak, and E. Ziegler, Appl. Phys. Lett. 81, 3287 (2002).

${ }^{27}$ A. Momose, S. Kawamoto, I. Koyama, Y. Hamaishi, K. Takai, and Y. Suzuki, Jpn. J. Appl. Phys. 42, L866 (2003).

${ }^{28}$ M. V. Berry and S. Klein, J. Mod. Opt. 43, 2139 (1996).
${ }^{29}$ T. Weitkamp, A. Diaz, C. David, F. Pfeiffer, M. Stampanoni, P. Cloetens, and E. Ziegler, Opt. Express 13, 6296 (2005).

${ }^{30}$ F. Pfeiffer, T. Weitkamp, O. Bunk, and C. David, Nat. Phys. 2, 258 (2006).

${ }^{31}$ M. Engelhardt, J. Baumann, M. Schuster, C. Kottler, F. Pfeiffer, O. Bunk, and C. David, Appl. Phys. Lett. 90, 224101 (2007).

${ }^{32}$ A. Olivo and R. Speller, Phys. Med. Biol. 52, 6555 (2007).

${ }^{33}$ A. Olivo, F. Arfelli, G. Cantatore, R. Longo, R. H. Menk, S. Pani, M. Prest, P. Poropat, L. Rigon, G. Tromba, E. Vallazza, and E. Castelli, Med. Phys. 28, 1610 (2001).

${ }^{34}$ A. Olivo and R. Speller, "Phase Contrast Imaging," International Patent WO/2008/029107 (13 March 2008).

${ }^{35}$ A. Olivo and R. Speller, Appl. Phys. Lett. 91, 074106 (2007).

${ }^{36}$ A. Olivo and R. Speller, Phys. Med. Biol. 53, 6461 (2008).

${ }^{37}$ A. Olivo, K. Ignatyev, P. R. T. Munro, and R. D. Speller, Appl. Opt. 50, 1765 (2011)

${ }^{38}$ A. Olivo, K. Ignatyev, P. R. T. Munro, and R. D. Speller, Nucl. Instrum. Methods A 610, 604 (2009).

${ }^{39}$ A. Olivo, L. Rigon, F. Arfelli, G. Cantatore, R. Longo, R. H. Menk, S. Pani, M. Prest, P. Poropat, G. Tromba, E. Vallazza, and E. Castelli, Med. Phys. 27, 2609 (2000).

${ }^{40}$ A. Olivo and R. Speller, Phys. Med. Biol. 54, N347 (2009).

${ }^{41}$ J. C. Buckland-Wright, Br. J. Radiol. 62, 201 (1989).

${ }^{42}$ A. Olivo and R. Speller, Phys. Med. Biol. 51, 3015 (2006).

${ }^{43}$ P. R. T. Munro, K. Ignatyev, R. D. Speller, and A. Olivo, Phys. Med. Biol. 55, 4169 (2010) 